\title{
The diffractionator
}

\section{Jack Gaskill, Craig Curtis}

Jack D. Gaskill, Craig H. Curtis, "The diffractionator," Proc. SPIE 2525, 1995 International Conference on Education in Optics, (13 October 1995); doi: 10.1117/12.224014

SPIE Event: SPIE's 1995 International Symposium on Optical Science, SPIE. Engineering, and Instrumentation, 1995, San Diego, CA, United States 
The diffractionator

Jack D. Gaskill

The University of Arizona, Optical Sciences Center

and Department of Electrical and Computer Engineering

Tucson, Arizona 85721

Craig H. Curtis

The University of Arizona, Optical Science Center

Tucson, Arizona 85721

\begin{abstract}
Physical demonstrations of diffraction and image formation for educational purposes have long been hampered by limitations of equipment and viewing facilities: it has usually been possible to demonstrate diffraction and image formation for only a few simple apertures or objects; it has often been time consuming to set up the optical bench used for the demonstration and difficult to keep it aligned; a darkened demonstration room has normally been required; and, it has usually been possible for only small groups of people to view the diffraction patterns and images. In 1990, the Optical Sciences Center was awarded an AT\&T Special Purpose Grant to construct a device that would allow diffraction and image formation demonstrations to be conducted while avoiding the limitations noted above. This device, which was completed in the fall of 1992 and is affectionately called "The Defractionator", makes use of video technology to permit demonstrations of diffraction, image formation and spatial filtering for large audiences in regular classrooms or auditoria. In addition, video tapes of the demonstrations can be recorded for viewing at sites where use of the actual demonstrator is inconvenient. A description of the system will be given, and video tapes will be used to display previously recorded diffraction phenomena and spatial filtering demonstrations.
\end{abstract}

Keywords: diffraction demonstration, Fraunhofer diffraction, Fresnel diffraction, spatial filtering, imaging demonstration

\title{
1. INTRODUCTION
}

Fourier theory has found application in many areas of science and engineering, but perhaps nowhere has it found a more natural home than in the field of optics. Why? Because optics, by its very nature, allows the utility of this incredible mathematical tool to be viewed directly. Such a unique characteristic serves as a powerful educational aid for those in the process of learning about the field. To illustrate, the spatial-frequency spectral composition of a monochromatic optical wavefield can be observed visually by viewing the Fraunhofer diffraction pattern of that wavefield ${ }^{1}$. Yet another demonstration helps explain the spatial-frequency performance of an optical imaging system. As a result, optics provides a natural home for the use of Fourier techniques. In contrast, while Fourier theory remains an extremely powerful tool in areas other than optics, its consequences can normally only be imagined or, at best, observed indirectly.

\section{DIFFRACTION}

"The Diffractionator", which makes use of video technology to permit demonstrations of diffraction for large audiences in regular classrooms or auditoria, has prooved to be a valuable tool in assisting students in their studies of this fascinating subject. Photographs of "The Diffractionator" are shown in Figs. 1 and 2, and the layout of the system is shown in Fig. 3. A diode laser, followed by a spatial filter/beam expander, is used to illuminate various diffracting objects that are placed at the location indicated in Fig. 3. The CCD video camera is then moved along the optical axis of the system, which allows sequential viewing of these diffracting objects, their associated Fresnel patterns, and the "Fraunhofer plane" on a video monitor. In this paper, we attempt to exhibit the utility of the instrument by including photographs of various diffracting objects, their associated Fresnel and Fraunhofer diffraction patterns and, in some cases, irradiance profiles of these patterns. The images were obtained by directly photographing the screen of a video monitor on which the patterns were displayed; consequently, although the purpose was served, the quality of the photographs is not as good as it might have been had other photographic techniques been used to record the images. 


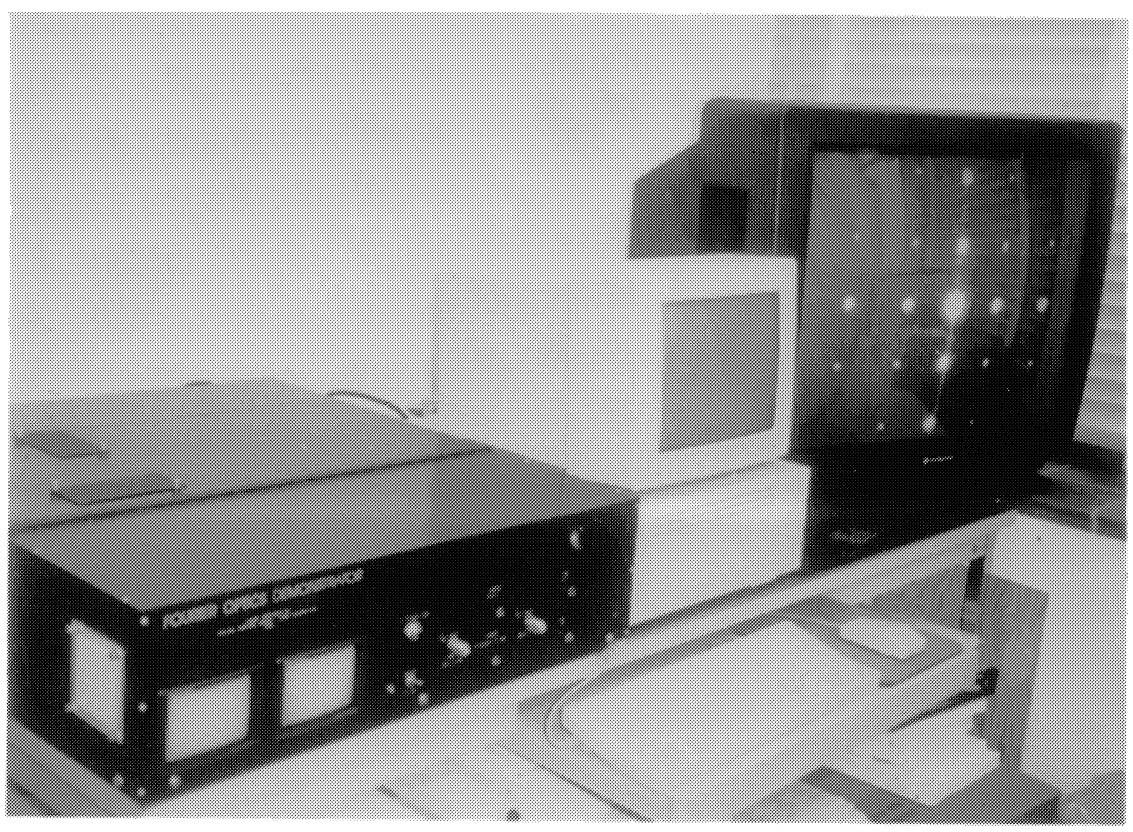

Fig. 1. Photograph of "The Diffractionator", computer and video monitor.

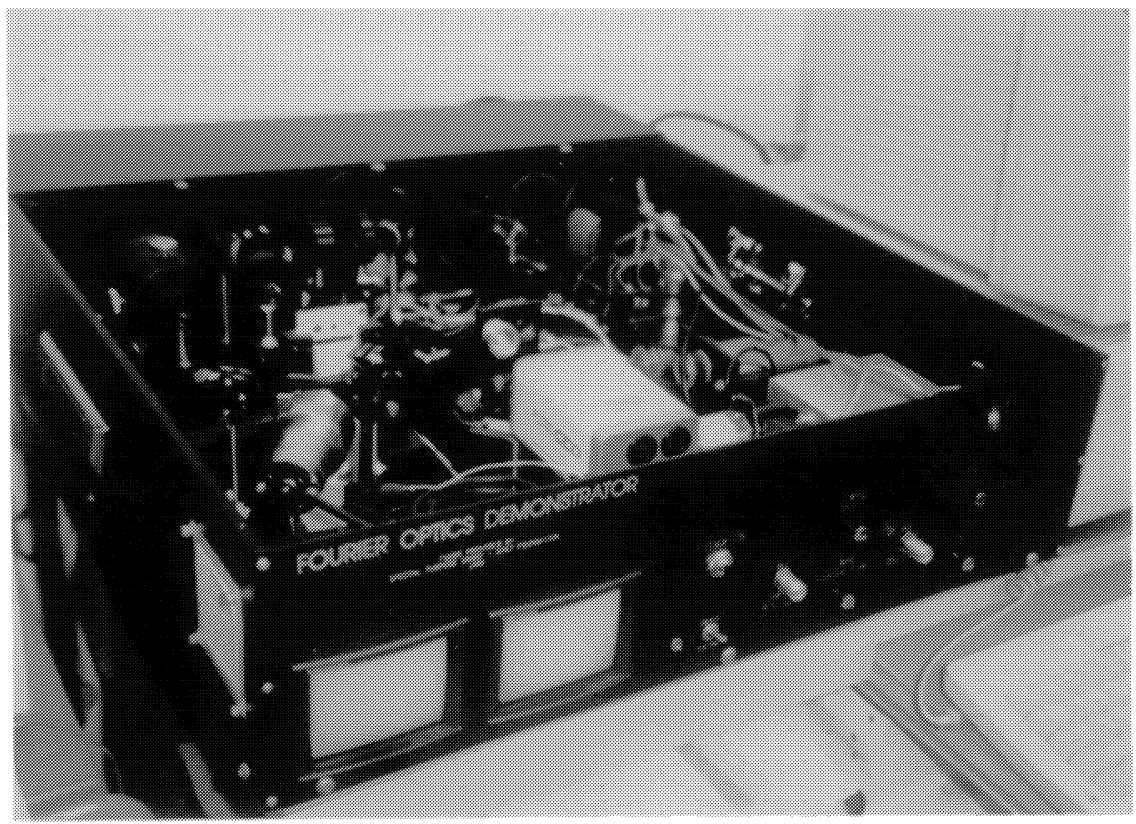

Fig. 2. Close-up view of "The Diffractionator" with cover removed. 


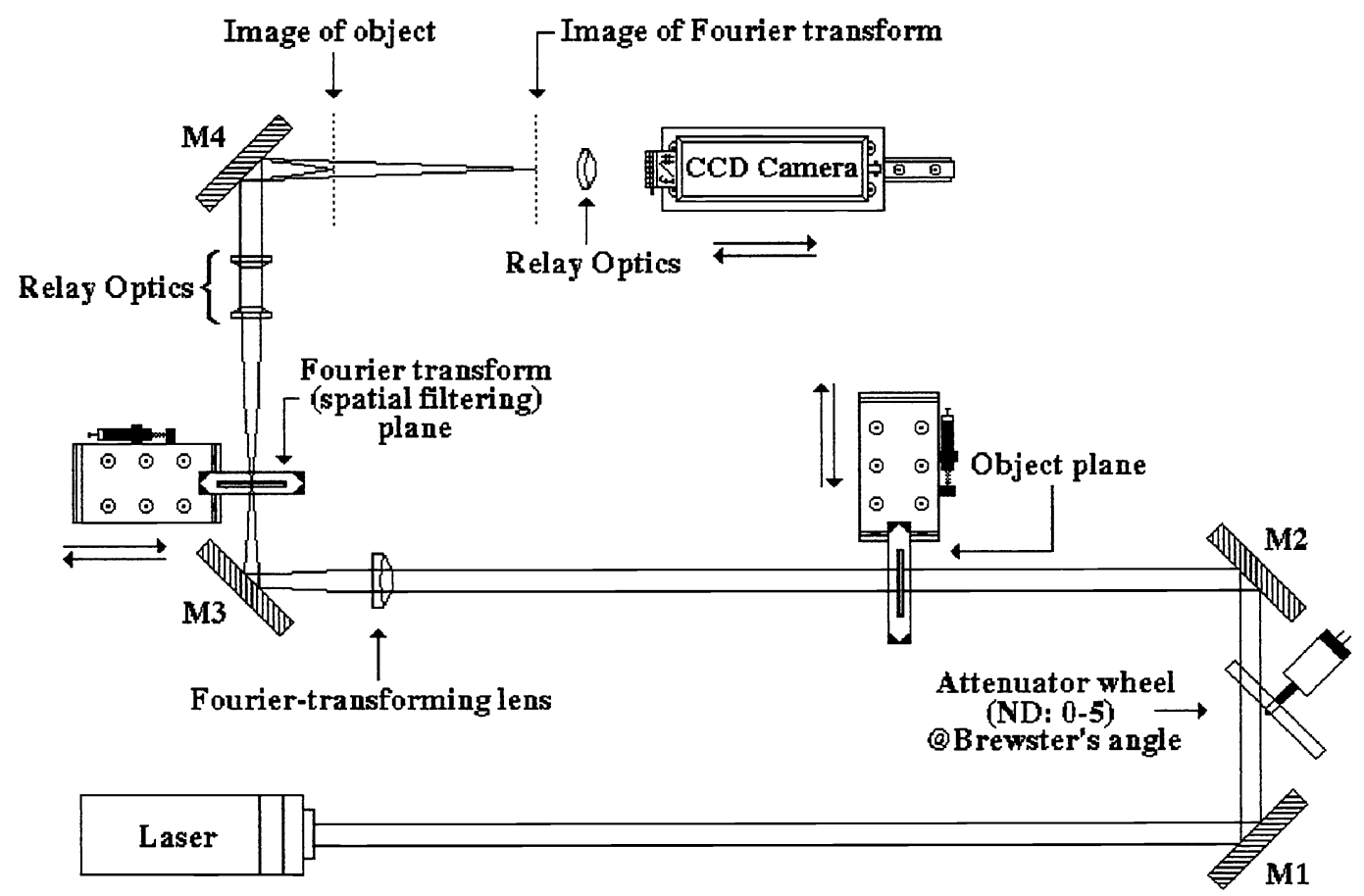

Fig. 3. System layout of "The Diffractionator".

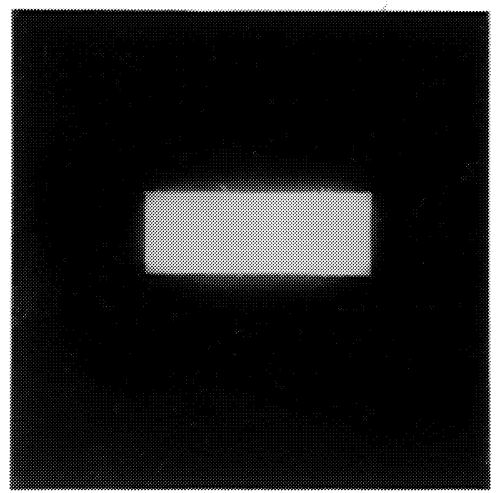

(a)

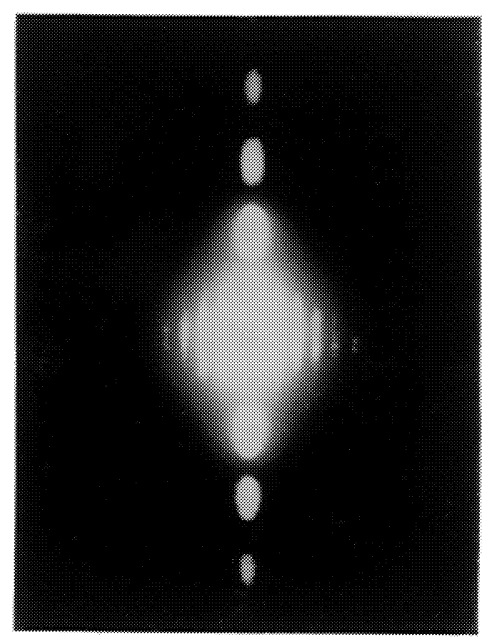

(b)

Fig. 4. Rectangular diffracting object (a) and its Fraunhofer diffraction pattern (b). 


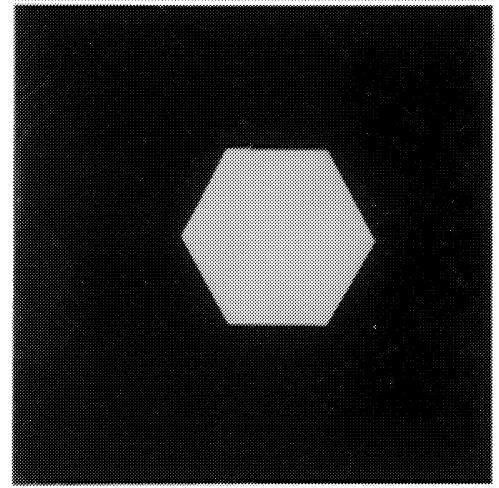

(a)

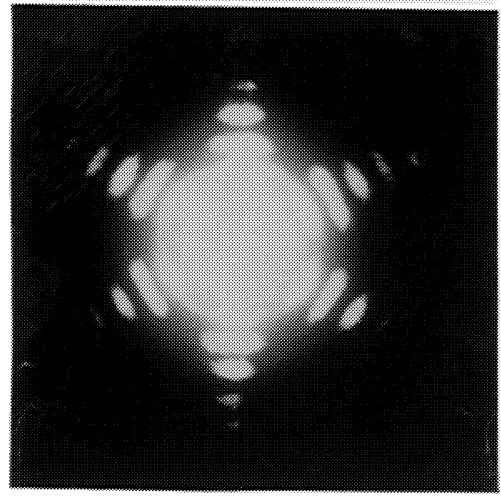

(b)

Fig. 5. Hexagonal diffracting object (a) and its Fraunhofer diffraction pattern (b).

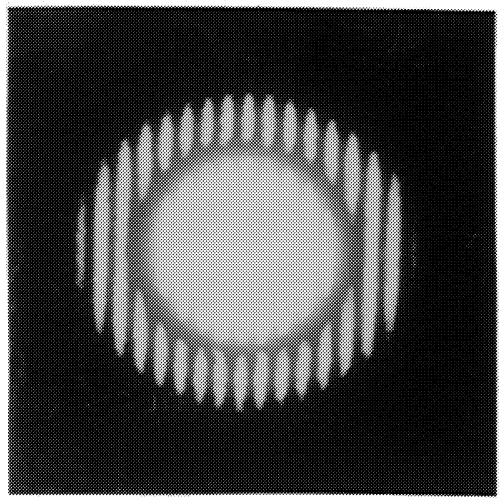

(a)

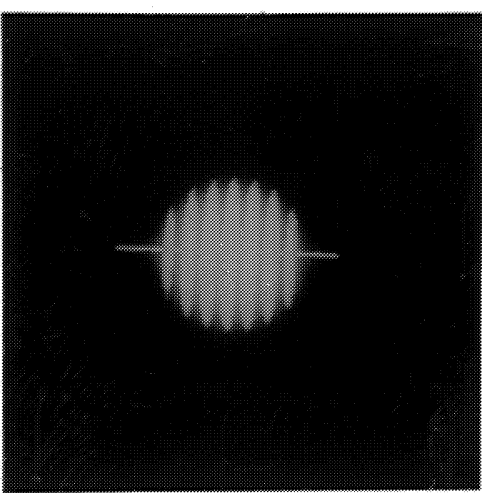

(b)

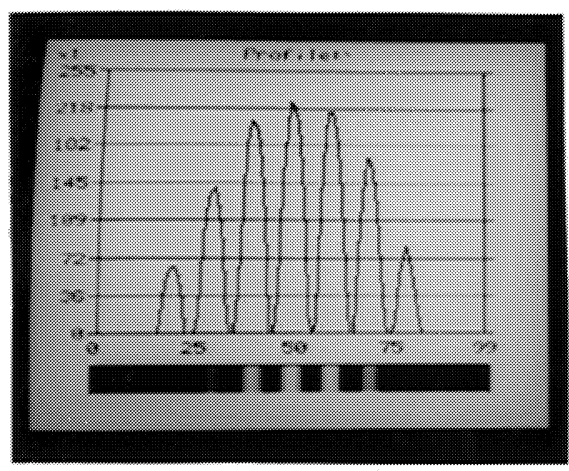

(c)

Fig. 6. Fraunhofer diffraction pattern of two circular apertures (a); the central part of this pattern (b); and, the irradiance profile along the line shown in the middle photograph (c)..

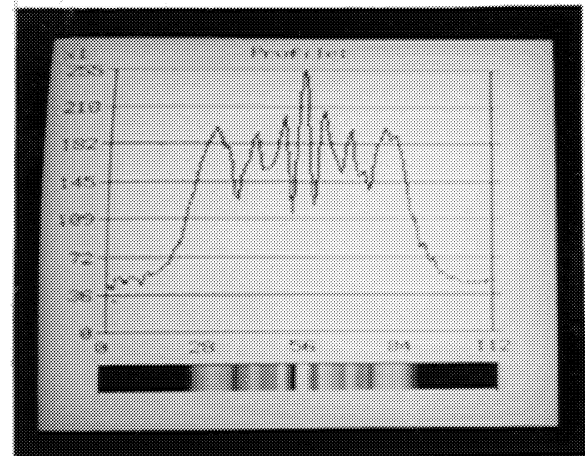

(a)

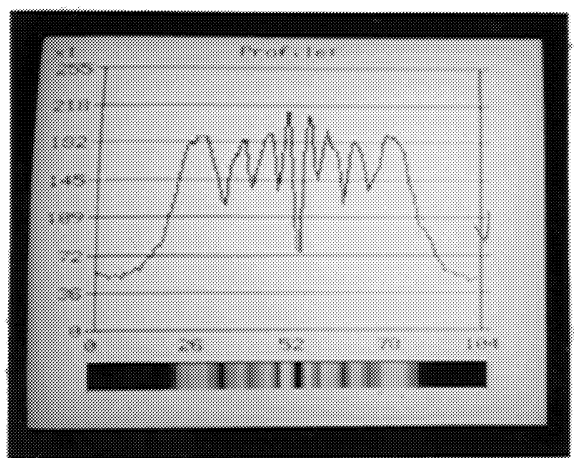

(b)

Fig. 7. Irradiance profiles across two Fresnel diffraction patterns of a circular diffracting object, first showing a central peak (a), and next showing a central minimum (b). 


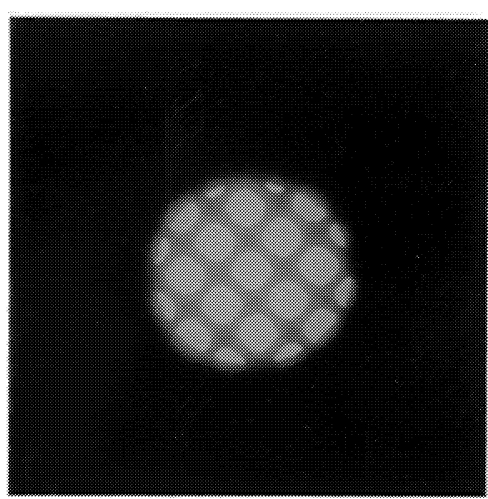

(a)

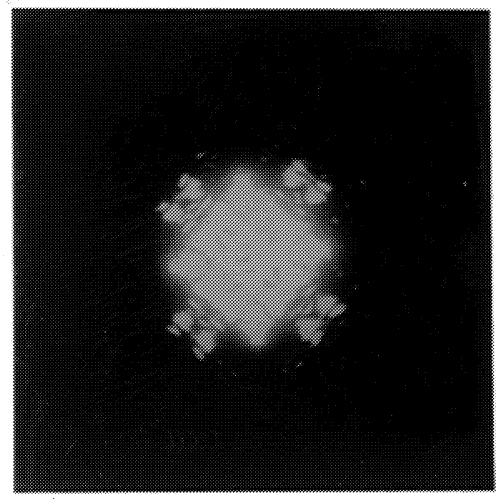

(d)

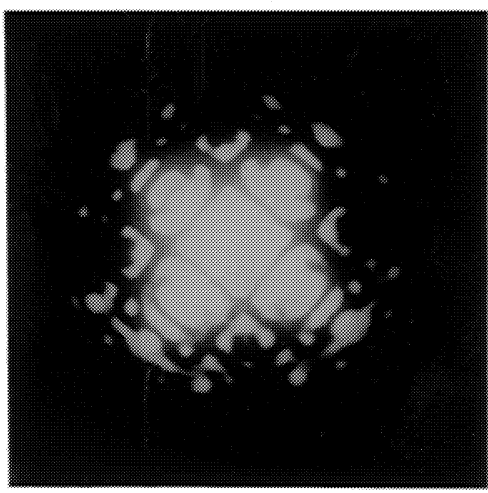

(g)

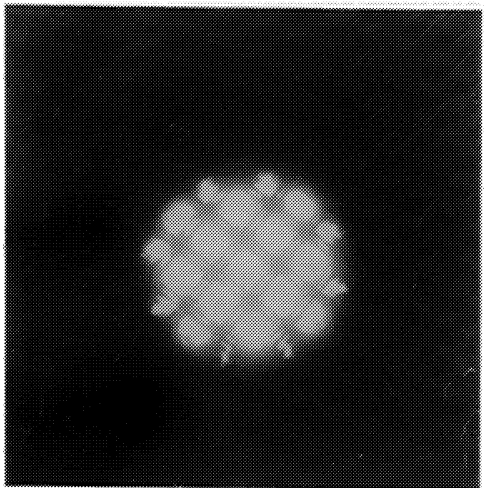

(b)

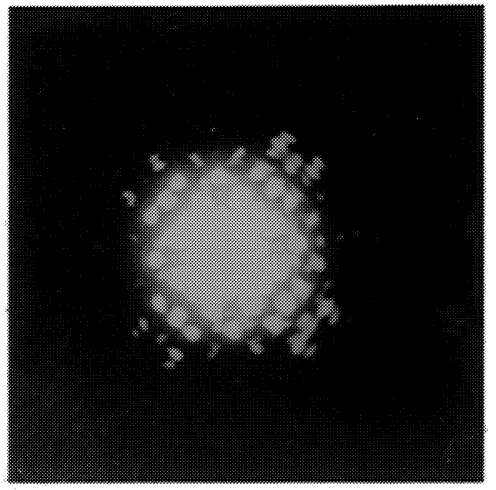

(e)

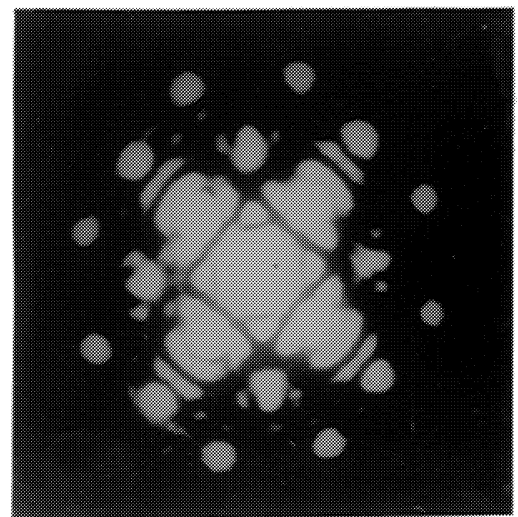

(h)

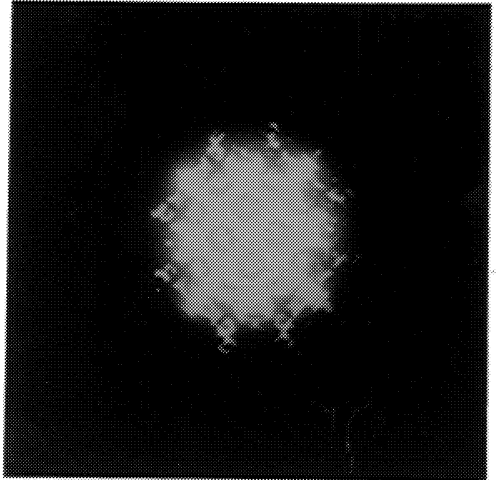

(c)

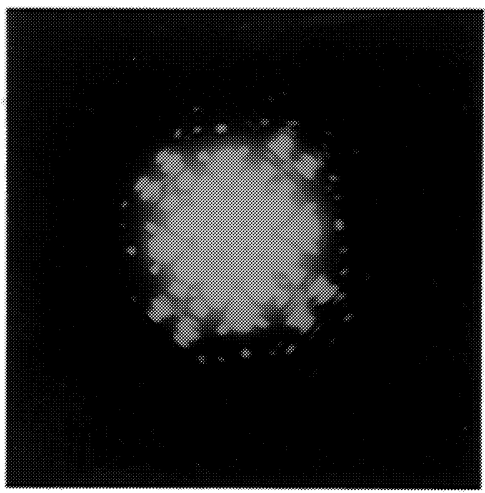

(f)

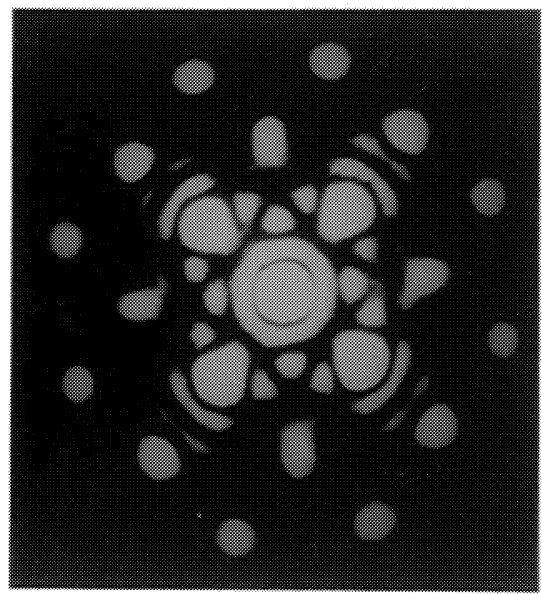

(i)

Fig. 8. Array-type diffracting object (a); Fresnel diffraction patterns as propagation distance increases (b) through (h); and, Fraunhofer diffraction pattern (i). 


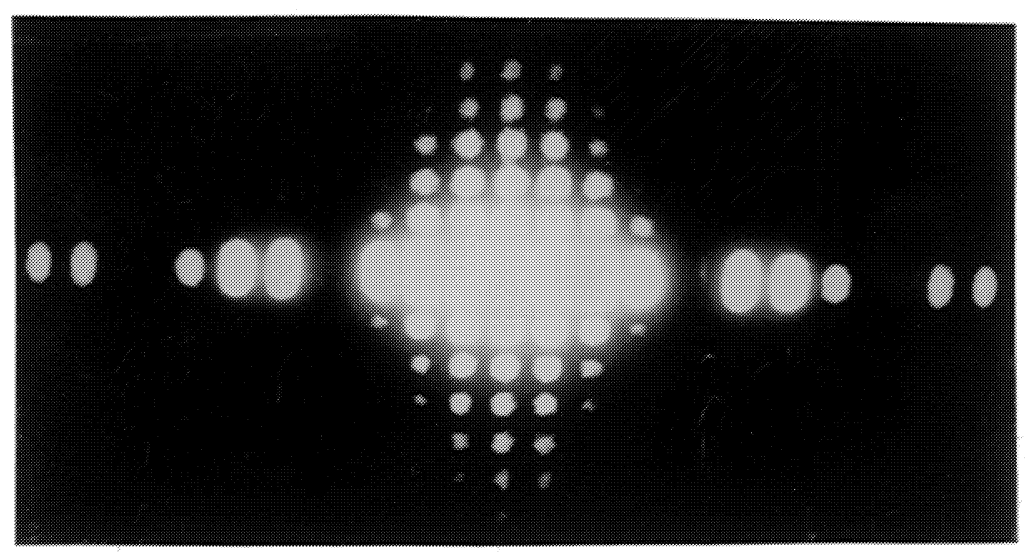

(a)

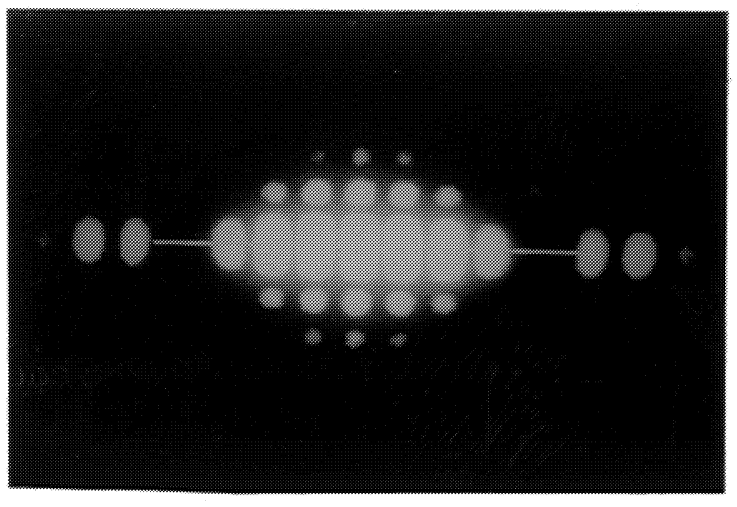

(b)

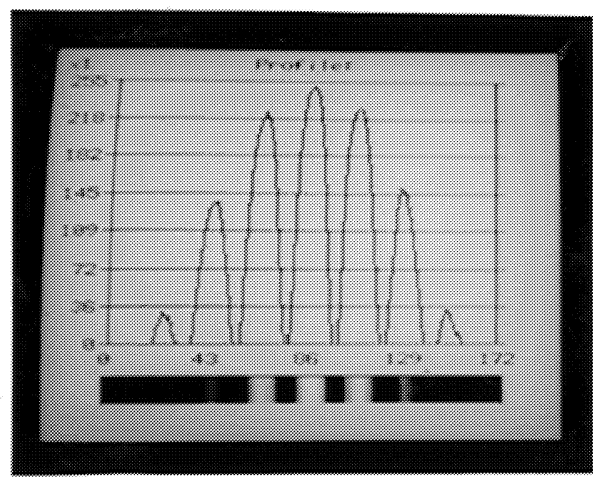

(c)

Fig. 9. Fraunhofer diffraction pattern of two rectangular apertures (a); the central part of this pattern (b); and, the irradiance profile along the line shown in the central part (c).

\section{SPATIAL FILTERING}

Let us now consider an ideal two-element system configured for imaging with coherent light ${ }^{1}$. The quality of an image produced by such a system is related to a number of characteristics, but perhaps central in importance is the aperture stop. When imaging with coherent light, the first lens element of the system causes the Fraunhofer diffraction pattern of the object to impinge upon the plane of the aperture stop, which then allows only a portion of the incident wavefield to be transmitted. The remaining lens element subsequently forms a Fraunhofer diffraction pattern of this transmitted light at the location of the image plane. Because the Fraunhofer pattern incident on the aperture stop represents a frequency-domain description of the object wavefield, because the wavefield transmitted by the stop represents the frequency-domain description of the image wavefield, and because the amplitude of the wavefield transmitted by the stop is described as a product of the incident wavefield amplitude and the amplitude transmittance of the stop, the stop effectively performs the role of a spatial filter. By placing various aperture stops, or spatial filters, at the "Fraunhofer plane" indicated in Fig. 3, the effects of diffraction on image formation can be demonstrated ${ }^{1}$. A fine rectangular screen, shown in Fig. 10 along with its Fraunhofer diffraction pattern, is imaged by a system having a slit-type aperture stop shown in Fig. 11. The spectral components passed by this spatial filter and the resulting image are shown in Fig. 12. Note that all of the Fourier components that contribute to horizontal structure in the object are filtered out, leaving only vertical structures. 


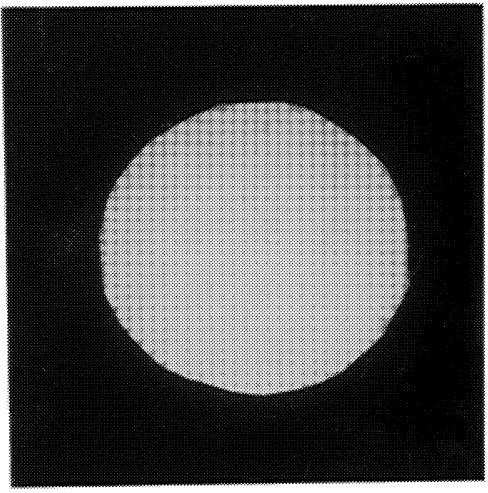

(a)

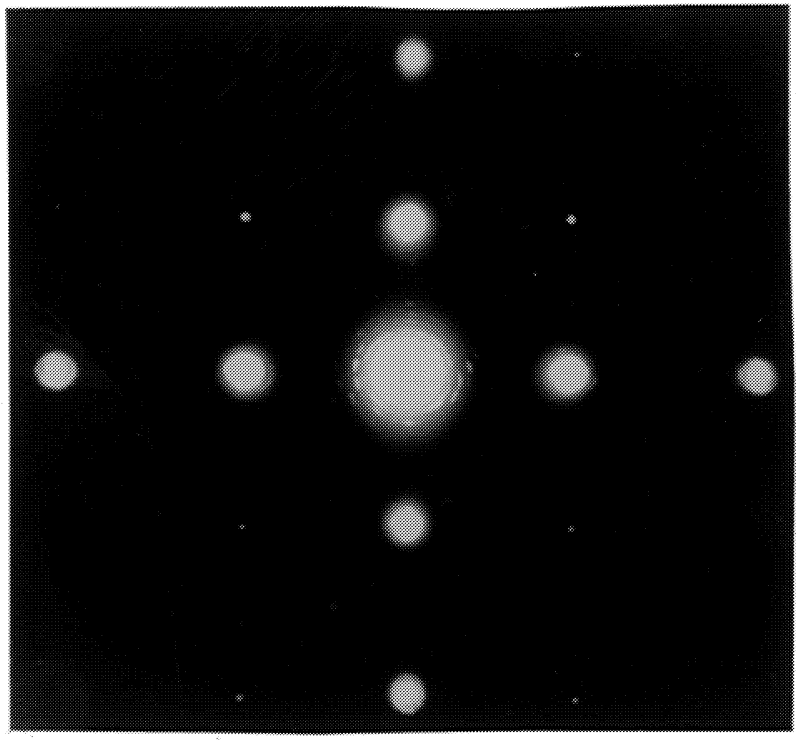

(b)

Fig. 10. Fine-screen object to be imaged (a), and its Fraunhofer diffraction pattern (b).

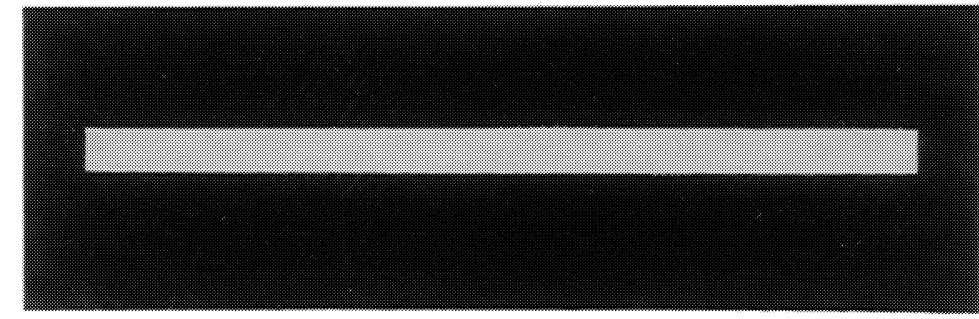

Fig. 11. Long narrow slit aperture stop of imaging system.

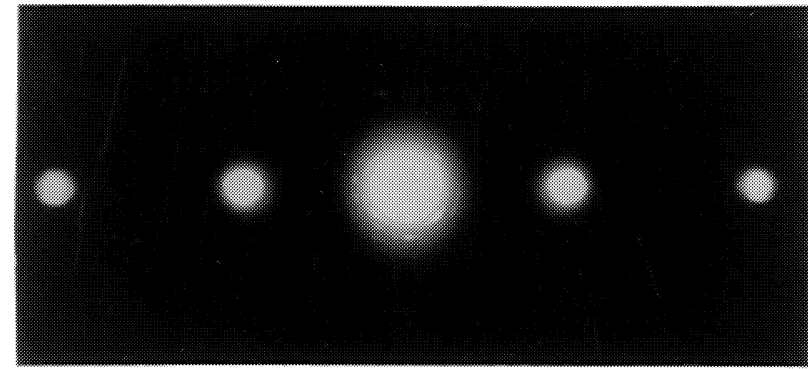

(a)

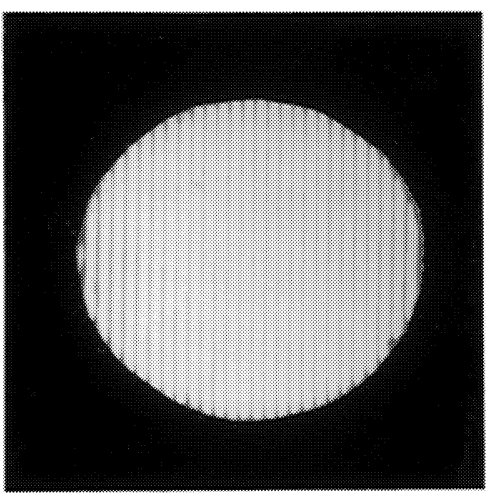

(b)

Fig. 12. Filtered spatial-frequency spectum of image (a), and resulting image showing no horizontal structure (b). 


\section{ACKNOWLEDGEMENT}

We gratefully acknowledge the AT\&T Special Purpose Grant that provided the funds necessary to construct "The Diffractionator."

\section{REFERENCES}

1. J. D. Gaskill, Linear Systems, Fourier Transforms, and Optics, Wiley, New York, 1978. 